\title{
The overview of the ALPACA Experiment
}

T. Asaba, ${ }^{a}$ K. Hibino, ${ }^{b}$ N. Hotta, ${ }^{c}$ M. Kataoka,${ }^{a}$ Y. Katayose,${ }^{a}$ C. Kato, ${ }^{d}$ K. Kawata, ${ }^{e}$ H. Kojima, ${ }^{f g}$ R. Mayta, ${ }^{h}$ P. Miranda, ${ }^{i}$ K. Munakata, ${ }^{d}$ Y. Nakamura, ${ }^{d}$ M. Nishizawa, ${ }^{j}$ S. Ogio, ${ }^{h}$ M. Ohnishi ${ }^{*},{ }^{e}$ A. Oshima, ${ }^{k}$ M. Raljevich, ${ }^{i}$ H. Rivera, ${ }^{i}$ T. Saito, ${ }^{l}$ T. K. Sako, ${ }^{m e}$ T. Sasaki, ${ }^{a}$ S. Shibata, ${ }^{k}$ A. Shiomi, ${ }^{n}$ M. Subieta, ${ }^{i}$ M. Suzuki, ${ }^{a}$ N. Tajima, ${ }^{o}$ M. Takita,${ }^{e}$ Y. Tameda, ${ }^{p}$ K. Tanaka, ${ }^{q}$ R. Ticona, ${ }^{i}$ H. Tsuchiya, ${ }^{r}$ Y. Tsunesada, ${ }^{h}$ S. Udo ${ }^{b}$ and M. Wakamatsu ${ }^{a}$ (The ALPACA Collaboration)

${ }^{a}$ Faculty of Engineering, Yokohama National University, Japan

${ }^{b}$ Faculty of Engineering, Kanagawa University, Japan

${ }^{c}$ Faculty of Education, Utsunomiya University, Japan

${ }^{d}$ Department of Physics, Shinshu University, Japan

${ }^{e}$ Institute for Cosmic Ray Research, The University of Tokyo, Japan

${ }^{f}$ Faculty of Engineering, Aichi Institute of Technology, Japan

${ }^{g}$ Chubu Innovative Astronomical Observatory, Japan

${ }^{h}$ Graduate School of Science, Osaka City University, Japan

${ }^{i}$ Instituto de Investigaciones Físicas, Universidad Mayor de San Andrés, Bolivia

${ }^{j}$ National Institute of Informatics, Japan

${ }^{k}$ College of Engineering, Chubu University, Japan

${ }^{l}$ Tokyo Metropolitan College of Industrial Technology, Japan

${ }^{m}$ Escuela de Ciencias Físicas y Nanotechnología, Yachay Tech, Ecuador

${ }^{n}$ College of Industrial Technology, Nihon University, Japan

${ }^{o}$ RIKEN, Japan

${ }^{p}$ Faculty of Engineering, Osaka Electro-Communication University, Japan

${ }^{q}$ Graduate School of Information Sciences, Hiroshima City University, Japan

${ }^{r}$ Japan Atomic Energy Agency, Japan

The ALPACA experiment is a new project aimed at wide field-of-view high-sensitivity observations of high-energy cosmic rays and cosmic gamma rays, launched between Bolivia and Japan in 2016. It is composed of an $83,000 \mathrm{~m}^{2}$ air shower array and a 5,400 $\mathrm{m}^{2}$ underground muon detector array, on a highland at the altitude of 4,740 m, halfway up Mount Chacaltaya on the outskirts of La Paz, Bolivia.

35th International Cosmic Ray Conference - ICRC2017

10-20 July, 2017

Bexco, Busan, Korea

${ }^{*}$ Speaker. 


\section{Introduction}

In the southern celestial hemisphere, the H.E.S.S. group reported the discovery of 14 new gamma-ray sources by the galactic plane survey [U]. Surprisingly, most of them were Unidentified (UNID) sources and faint in X-rays or other wavelengths. As the H.E.S.S. survey was limited within the galactic plane in the southern celestial hemisphere due to its narrow field of view, the importance of a wide field-of-view unbiased survey is emphasized. Furthermore, many of the 14 sources have a harder energy spectrum at $\mathrm{TeV}$ energies (indices: -1.8 to -2.8 ) than the standard Crab candle (index; -2.6). Although, the energy spectra were mostly measured below approximately $10 \mathrm{TeV}$ due to the sensitivity limit of the H.E.S.S. air Cherenkov telescopes, they suggest that they extend up to the $100 \mathrm{TeV}$ region. The number of $\mathrm{TeV}$ sources now amounts to approximately 200. However, the origin of the cosmic rays has not yet been clarified.

Cosmic rays are supposed to be accelerated up to the knee energy $(\mathrm{PeV})$ region at supernova remnants (SNRs) in our galaxy. Therefore, we naturally expect gamma rays at $100 \mathrm{TeV}$ energies, which originate in $\pi^{0}$ decays produced by the accelerated cosmic rays interacting with matter surrounding the SNRs. However, on-going experiments focus on measuring gamma rays in the $1-10 \mathrm{TeV}$ region. The gamma-ray emission of electron origin might be highly suppressed above $10 \mathrm{TeV}$ due to the rapid decrease of the inverse-Compton cross section by the Klein-Nishina effect as well as synchrotron radiation energy losses in the strong magnetic field around SNRs.

The detection and spectral measurement of gamma rays in the $100 \mathrm{TeV}$ region from their celestial sources, together with multi-wavelength (radio, X-ray, gamma-ray) observations, will be an important experiment enabling us to discriminate between the two processes (cosmic-ray / electron origins), to locate the acceleration site of cosmic rays (PeVatrons which accelerate cosmic rays up to $\mathrm{PeV}$ energies) and to verify the standard acceleration model. Some of the H.E.S.S. 14 sources are very promising candidates for the cosmic-ray PeVatron. Furthermore, the energy spectrum of diffuse gamma rays from the extended region around the galactic center marginally measured by H.E.S.S. up to approximately $10 \mathrm{TeV}$ also strongly indicates the existence of PeVatrons [ฉ], from which we expect to detect gamma rays at $100 \mathrm{TeV}$ energies. Thus, a wide field-of-view gamma-ray imaging at $100 \mathrm{TeV}$ energies in the southern sky, where the H.E.S.S. sources, the Fermi bubbles and the galactic center are located within the field of view, will be a key experiment.

\section{Experiment}

The experimental site for the ALPACA (Andes Large area PArticle detector for Cosmic ray physics and Astronomy) project is located on a plateau called Chacaltaya Hill (4,740 m above sea level, $16^{\circ} 23^{\prime} \mathrm{S}, 68^{\circ} 08^{\prime} \mathrm{W}$ ), as shown in Fig. 四, around Mount Chacaltaya, near La Paz, Bolivia. This plateau is a flat and horizontal land covering the area of more than $500 \mathrm{~m} \times 500 \mathrm{~m}$. Our detectors will be set up in some part of this area.

We plan to set up a 5,400 $\mathrm{m}^{2}$ underground (approximately one to a few meters) muon detector array (MD) and an 83,000 $\mathrm{m}^{2}$ air shower array (AS), shown in Fig. [ [ [B]]. The MD of the water Cherenkov type is composed of eight pools with each pool (approximately $1 \mathrm{~m}$ deep) containing twelve $56 \mathrm{~m}^{2}$ unit detectors. AS is made up of $4011.0 \mathrm{~m}^{2}$ plastic scintillation counters at $15 \mathrm{~m}$ spacing. 


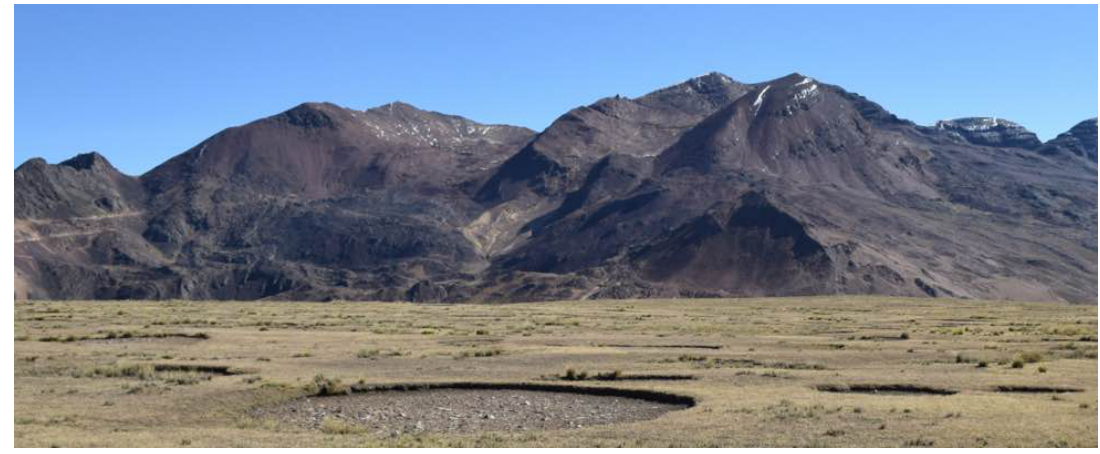

Figure 1: Experimental site for the ALPACA experiment, Chacaltaya Hill $(4,740 \mathrm{~m}$ above see level, $\left.16^{\circ} 23^{\prime} \mathrm{S}, 68^{\circ} 08^{\prime} \mathrm{W}\right)$, near Mount Chacaltaya, in Bolivia.

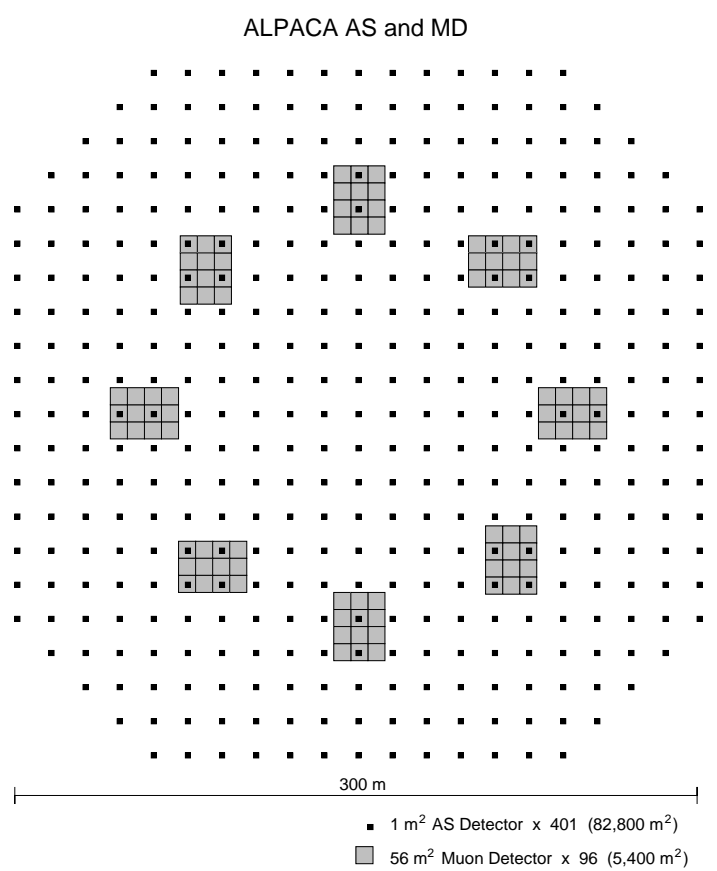

Figure 2: Schematic view of the ALPACA experiment. The small black squares indicate $4011.0 \mathrm{~m}^{2}$ plastic scintillator detectors, forming an air shower array with $83,000 \mathrm{~m}^{2}$ in area. The grey rectangles indicate eight underground muon detector pools, each of which contains twelve $56 \mathrm{~m}^{2}$ muon detector units. The total area of the underground muon detector array is $5,400 \mathrm{~m}^{2}$.

The AS field of view is roughly 2 steradians. The expected angular resolution of AS is approximately 1 degree at $5 \mathrm{TeV}$ and 0.2 degrees around $100 \mathrm{TeV}$ for gamma rays. For $100 \mathrm{TeV}$ gamma rays, the AS energy resolution is estimated to be $\sim 25 \%$. The hadron rejection power of MD is more than $99.9 \%$ at $100 \mathrm{TeV}$, while keeping most of gamma-ray events. The long-term detector stability, angular resolution, pointing accuracy and energy scale can be calibrated by the cosmic ray shadow of the Moon as well as by some of the bright stable $\mathrm{TeV}$ gamma ray sources in the southern sky. 


\section{Physics Targets}

We have four physics targets in the ALPACA experiment:

1. Measurement of high-energy $(5 \mathrm{TeV}-1 \mathrm{PeV})$ cosmic gamma rays.

2. Measurement of cosmic ray energy spectra around the Knee energy region (100 TeV $100 \mathrm{PeV})$.

3. Measurement of cosmic ray anisotropy $>5 \mathrm{TeV}$ in the sidereal time frame.

4. Measurement of the Sun shadow in cosmic rays $>5 \mathrm{TeV}$.

We aim at low-background detection of celestial gamma rays in the $100 \mathrm{TeV}$ region with a world-best sensitivity (an order of magnitude better than any previous / existing experiments) and at locating the origins of cosmic rays accelerated up to the knee energy region in the southern sky. Assuming the presence of a Crab-like gamma-ray source in the southern sky, with a power-law differential energy spectrum proportional to $E^{-2.6}$ extending up above $100 \mathrm{TeV}$ where $E$ is energy of a gamma ray, the ALPACA experiment is sensitive to the source with $\sim 15 \%$ Crab intensity during one calendar year, as demonstrated in Fig. [].

The ALPACA experiment will be a unique experiment complementary to on-going experiments (FERMI, H.E.S.S., VERITAS, MAGIC, CALET, Tibet AS $\gamma$, HAWC) and future projects (LHAASO, CTA) in this field, which are either located in the northern hemisphere or aiming at gamma-ray astronomy below $10 \mathrm{TeV}$ region, or having a narrow field-of-view. Thus, the new energy window in the $100 \mathrm{TeV}$ region observing gamma rays with a wide field-of-view will be opened first in the southern sky by the ALPACA experiment. We expect to detect more than a dozen established sources, i.e., young SNRs (SN 1006 Remnant, RX J1713.7 - 3946, RX J0852.0 - 4622), Pulsar Wind Nebulae, the galactic center etc. in the $100 \mathrm{TeV}$ region, some of which may be

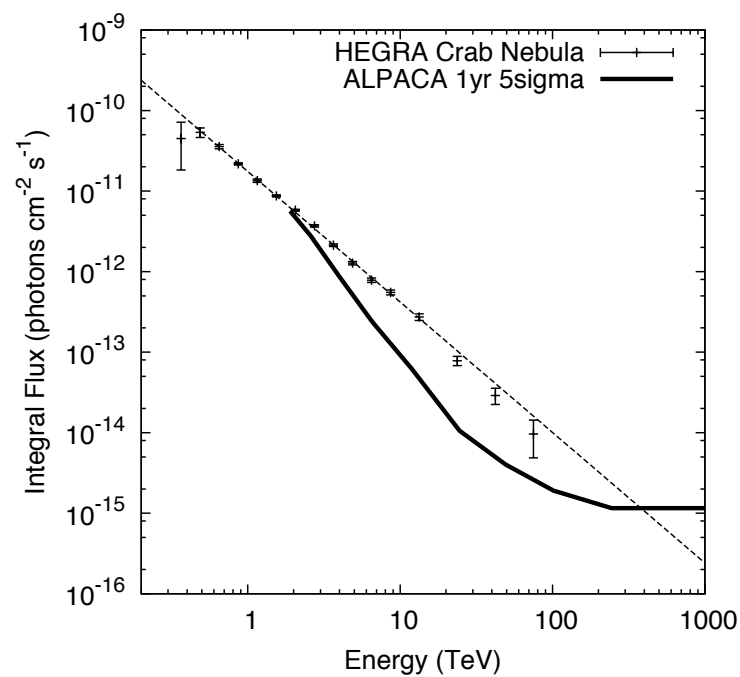

Figure 3: Sensitivity of ALPACA to high-energy gamma-ray point sources. The experimental points are from Ref. [四]. The ALPACA sensitivity is evaluated from Ref. [回]. 
cosmic-ray PeVatron candidates. Furthermore, our wide field-of-view sensitivity to diffuse gamma rays allows us to study extremely diffuse gamma-ray sources which are difficult to detect by IACTs. The diffuse gamma rays from the Fermi bubbles recently reported by the Fermi-LAT group may be clearly detected, if they extend up to the $100 \mathrm{TeV}$ region. Similarly, the detection of diffuse gamma rays above $100 \mathrm{TeV}$ from an extended region from the galactic center is promising, where the gamma-ray energy spectrum strongly suggests the existence of PeVatrons. Detection and spectral measurement of gamma rays in the $100 \mathrm{TeV}$ region from these celestial sources, together with multi-wavelength observations (radio, X-ray, gamma-ray), are key points enabling us to discriminate between the two processes (cosmic-ray/electron origins), to locate the acceleration site of cosmic rays and to examine the standard acceleration model of cosmic rays.

From the astronomical point of view, we pioneer ultra-high energy gamma-astronomy (above $100 \mathrm{TeV}$ ) in the southern sky. Besides, gamma-ray emission from near-by extra-galactic sources, e.g. M87, Cen A, gamma rays of dark matter origin, those from the Sun disk recently observed by Fermi [ [G] may be interesting subjects.

We also aim to measure the energy spectra of proton, helium and iron components separately around the knee energy region with the ALPACA experiment. The standard cosmic-ray acceleration model at SNRs predicts the knee energy of each nucleus component being proportional to $Z$ (atomic number). We can discriminate proton and iron components by $\mathrm{MD}$, as an iron nucleus produces approximately twice as many muons as a proton with the same energy. Thus, the cosmic-ray acceleration scenario (SNR shock acceleration) will be verified by observing the linearly Z-dependent knee (=bent) positions of proton, helium, iron components around the knee energy region.

Precise cosmic-ray anisotropy measurements in the sidereal time frame in the $\mathrm{TeV}$ energy region in the southern sky provide unique data for the community to understand the magnetic field structure in the heliosphere. The ALPACA experiment gives complementary data in the $\mathrm{TeV}$ region to those from IceCube above a few tens of TeV.

Furthermore, measurements of the Sun shadow in cosmic rays above the TeV energy region in the southern hemisphere also helps understand the modeling of the magnetic fields between the Sun and the Earth, complementary to the observations in the northern hemisphere.

\section{Summary}

We have started up the ALPACA project which aims at imaging the southern sky by means of gamma rays in the $100 \mathrm{TeV}$ region, composed of a southern-hemisphere air shower array $\left(83,000 \mathrm{~m}^{2}\right)$ with a large $\left(5,400 \mathrm{~m}^{2}\right)$ underground water Cherenkov muon detector array in the Andes highland $(4,740 \mathrm{~m}$ above sea level) in Bolivia. The muon detector array enables us to discriminate the muon-poor gamma-ray component from the muon-rich hadronic component, allowing us to make low-background observations of gamma rays in the $100 \mathrm{TeV}$ region. This will be the first important attempt in the southern sky in this field. It should be stressed that, until now, there has not existed any high-sensitivity experiment capable of probing the gamma-ray southern sky around $100 \mathrm{TeV}$ with a wide field-of-view and high duty cycle.

The ALPACA experiment covers a wide range of cosmic ray physics, e.g., measurement of the chemical composition and energy spectra around the knee energy region, measurement of the 
cosmic ray anisotropy above the $\mathrm{TeV}$ energy region in the southern hemisphere, measurement of the Sun shadow in cosmic rays in the southern hemisphere etc.

Thus, the expected impacts upon the field are promising, as the project gives an important clue to the problem which has remained over 100 years since the discovery of cosmic rays: the origin, acceleration and propagation mechanism of cosmic rays.

\section{Acknowledgments}

The authors would like to thank the staff of the Instituto de Investigaciones Físicas, Universidad Mayor de San Andrés, La Paz, Bolivia, for their support to our experiment at Mount Chacaltaya. This work is supported by the joint research program of the Institute for Cosmic Ray Research (ICRR), The University of Tokyo. KK is supported by the Toray Science Foundation.

\section{References}

[1] F. Aharonian et al., A New Population of Very High Energy Gamma-Ray Sources in the Milky Way, Science, 307, 1938-1942 (2005)

[2] A. Abramoswski et al., Acceleration of petaelectronvolt protons in the Galactic Centre, Nature, 531, 476 (2016)

[3] M. Takita, The ALPACA Project, Proc. of 19th ISVHECRI (2016)

[4] F. Aharonian et al., The Crab Nebula and Pulsar between $500 \mathrm{GeV}$ and $80 \mathrm{TeV}$ : Observations with the HEGRA stereoscopic air Cherenkov telescopes, ApJ, 614, 897-913 (2004)

[5] T. K. Sako et al., Exploration of a $100 \mathrm{TeV}$ gamma-ray northern sky using the Tibet air-shower array combined with an underground water-Cherenkov muon-detector array, Astroparticle Physics, 32, 177-184 (2009)

[6] K. C. Y. Ng et al., First observation of time variation in the solar-disk gamma-ray flux with Fermi, Phys. Rev. D, 94, 023004 (2016) 